\title{
Reverse Regioselectivity in Reductive Ring Opening of Epoxide Ena- bled by Zirconocene and Photoredox Catalysis
}

\author{
Kazuhiro Aida, ${ }^{a}$ Marina Hirao, ${ }^{a}$ Aiko Funabashi, ${ }^{a}$ Natsuhiko Sugimura, ${ }^{b}$ Eisuke Ota, ${ }^{*} a$ and Junichiro \\ Yamaguchi*a $^{*}$ \\ ${ }^{a}$ Department of Applied Chemistry, Waseda University, 513 Wasedatsurumakicho, Shinjuku, Tokyo 162-0041, Japan. \\ ${ }^{b}$ Materials Characterization Central Laboratory, Waseda University, 3-4-1 Ohkubo, Shinjuku, Tokyo 169-8555, Japan
}

\section{KEYWORDS Zirconocene, Epoxide, Ring opening, Reverse regioselectivity, Photoredox catalysis}

ABSTRACT: A ring opening of epoxide with zirconocene and photoredox catalysis has been developed. Compared to the ring opening methods with titanocene, the present protocol exhibited reverse regioselectivity to afford more-substituted alcohols via putative less-stable radicals. The observed regioselectivity could be explained by shifting the transition states to more reactant-like structures by changing the metal center of the metallocene catalyst.

Epoxide is a common structural motif in bioactive compounds, naturally occurring feedstocks, and various synthetic intermediates. Due to their inherent ring strain, epoxides generally behave as an excellent electrophile. Contrastingly, homolysis of its $\mathrm{C}-\mathrm{O}$ bond provides a nucleophilic carbon radical leading to a distinct product that is unavailable from polar mechanisms. For such transformations, titanocene (III) $\left(\mathrm{Cp}_{2} \mathrm{TiX}\right)$ has been exclusively exploited over the past 30 years since its introduction by Nugent and RajanBabu. ${ }^{1,2}$ In general, an epoxide coordinates to titanocene (III) prepared by reduction of titanocene (IV) with a reducing metal, and the $\mathrm{C}-\mathrm{O}$ bond cleavage proceeds via single electron transfer (Figure 1A). The resulting carbon radical reacts with a large array of trapping agents such as hydrogen atom donors or olefins to furnish alcohols. So far, considerable research efforts have been directed to broaden this transformation. ${ }^{3-7}$ This has enabled remarkable advances in functionalization of the radical generated via epoxide ring opening, even including cross-coupling. ${ }^{8}$ Mild reaction conditions have allowed this transformation to contribute to numerous natural product syntheses.9-11 Recent studies showed that the merger of titanocene and photoredox catalysis can circumvent the need for a stoichiometric reducing metal. ${ }^{12-14}$

When an unsymmetrical epoxide is employed, regioselectivity issues must be addressed. The factors controlling the regioselectivity is a fascinating topic, and detailed studies have been carried out.15-17 In most cases, homolysis predominantly occurs at the $\mathrm{C}-\mathrm{O}$ bond, which gives a more stable radical (Figure 1B). In particular, thermodynamically stable radicals in benzylic ${ }^{18}$, allylic ${ }^{19}$, and anomeric ${ }^{20}$ positions are favorably generated, much like in many other radical reactions. For electronically unbiased epoxides, non-bonding interactions including steric hindrance are considered as a prevailing factor. ${ }^{15-17}$ However, the princi- ples controlling the regioselectivity still remain to be elucidated, and only few examples exist where the product is derived from a putative less stable radical. ${ }^{15,21}$

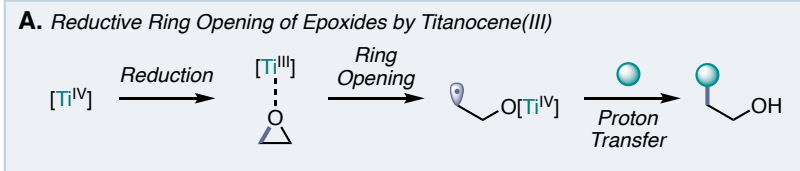

B. Conventional Regioselectivity in Ring Opening of Asymmetric Epoxides

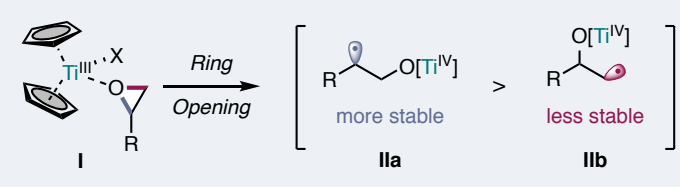

$\mathrm{C}-\mathrm{O}$ bond homolysis proceeds at the carbon to give more stable radical Challenges: Achievement of regioselectivity independent to radical stability

C. Altering Transition State of Ring Opening with Zirconocene

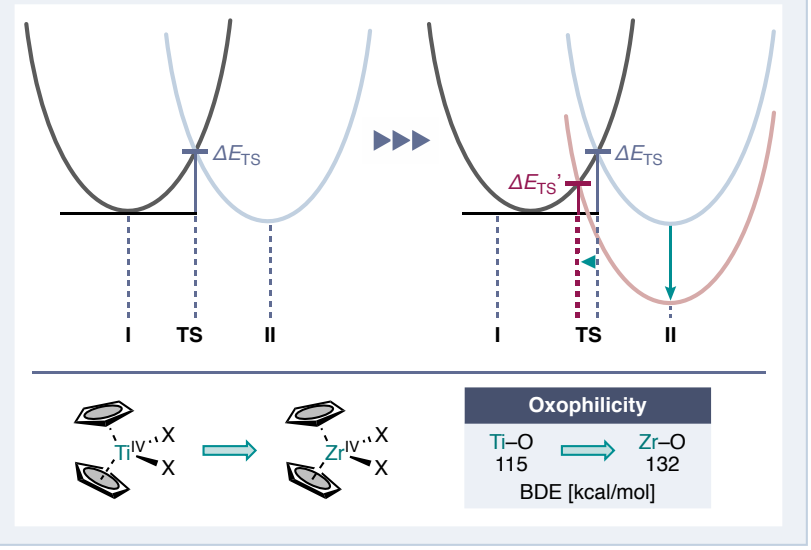

Figure 1. Reductive ring opening of epoxides (A) General scheme of titanocene(III)-catalyzed ring opening (B) Conven- 
tional regioselectivity. (C) Shifting transition state using zirconocene.

Considering the Bell-Evans-Polanyi principle, ${ }^{22,23}$ activation energy $\left(\Delta E_{\mathrm{TS}}\right)$ decreases if epoxide opening becomes more exothermic (Figure 1C). Likewise, the transition state would shift earlier and become more similar to the starting material. In an earlier transition state, the contribution of stability of the resulting radicals in regioselectivity would diminish according to the Hammond postulate. ${ }^{24}$ Inspired by the difference in bond dissociation energy (BDE) between Ti-0 (115 kcal/mol) ${ }^{25}$ and $\mathrm{Zr}-0(132 \mathrm{kcal} / \mathrm{mol}),{ }^{25}$ we became interested in using zirconocene instead of titanocene for the epoxide opening. Its stronger oxophilicity should render the ring opening dramatically exothermic. On this basis, we envisioned that a zirconocene-catalyzed ring opening of epoxide would reverse the conventional regioselectivity expected from relative radical stability.

\section{Table 1. Reaction Optimization}

\begin{tabular}{|c|c|c|c|c|}
\hline${ }_{\mathbf{1 A}}$ & $\begin{array}{c}5.0 \mathrm{~mol} \% \\
3.0 \mathrm{~mol} \% \\
60 \mathrm{~mol} \\
1,4-\mathrm{CHD} \\
\mathrm{PhCF}_{3} \\
\text { blue LEDs }\end{array}$ & $\begin{array}{l}\text { zirconocene } \\
\text { hotocatalyst } \\
\text { thiourea } \\
\text { (3.0 equiv) } \\
0.050 \mathrm{M}) \\
35^{\circ} \mathrm{C}, 12 \mathrm{~h}\end{array}$ & 1B & $1 \mathrm{C}$ \\
\hline entry & $\mathrm{Cp}_{2} \mathrm{ZrX}_{2}$ & photocatalyst & thiourea & 1B/1C $(\%)^{a}$ \\
\hline 1 & $\mathrm{Cp}_{2} \mathrm{ZrCl}_{2}$ & P1 & none & $0 / 0$ \\
\hline 2 & $\mathrm{Cp}_{2} \operatorname{Zr}(\mathrm{OTf})_{2} \cdot \mathrm{THF}$ & P1 & none & $0 / 0$ \\
\hline 3 & $\mathrm{Cp}_{2} \operatorname{Zr}(\mathrm{OTf})_{2} \cdot \mathrm{THF}$ & P1 & $\mathrm{T} 1$ & $34 / 7$ \\
\hline 4 & $\mathrm{Cp}_{2} \operatorname{Zr}(\mathrm{OTf})_{2} \cdot \mathrm{THF}$ & P1 & $\mathrm{T} 2$ & $63 / 5$ \\
\hline 5 & $\mathrm{Cp}_{2} \operatorname{Zr}(\mathrm{OTf})_{2} \cdot \mathrm{THF}$ & P1 & T3 & $79 / 3$ \\
\hline $6^{b}$ & $\mathrm{Cp}_{2} \operatorname{Zr}(\mathrm{OTf})_{2} \cdot \mathrm{THF}$ & P1 & $\mathrm{T} 3$ & $37 / 2$ \\
\hline $7^{c}$ & $\mathrm{Cp}_{2} \operatorname{Zr}(\mathrm{OTf})_{2} \cdot \mathrm{THF}$ & P1 & $\mathrm{T} 3$ & $47 / 26$ \\
\hline 8 & $\mathrm{Cp}_{2} \operatorname{Zr}(\mathrm{OTf})_{2} \cdot \mathrm{THF}$ & P2 & $\mathrm{T} 3$ & $77 / 7$ \\
\hline 9 & $\mathrm{Cp}_{2} \operatorname{Zr}(\mathrm{OTf})_{2} \cdot \mathrm{THF}$ & P3 & $\mathrm{T} 3$ & $58 / 2$ \\
\hline 10 & $\mathrm{Cp}_{2} \mathrm{ZrCl}_{2}$ & P1 & $\mathrm{T} 3$ & $69 / 7$ \\
\hline 11 & $\mathrm{Cp}_{2} \operatorname{Zr}(\mathrm{OTs})_{2}$ & P1 & $\mathrm{T} 3$ & $53 / 47$ \\
\hline $12^{d}$ & $\mathrm{Cp}_{2} \mathrm{Zr}(\mathrm{OTf})_{2} \cdot \mathrm{THF}$ & P1 & $\mathrm{T} 3$ & $80 / 3$ \\
\hline $13^{d}$ & none & P1 & T3 & $0 / 0$ \\
\hline $14^{d}$ & $\mathrm{Cp}_{2} \operatorname{Zr}(\mathrm{OTf})_{2} \cdot \mathrm{THF}$ & none & T3 & $0 / 0$ \\
\hline $15^{d, e}$ & $\mathrm{Cp}_{2} \operatorname{Zr}(\mathrm{OTf})_{2} \cdot \mathrm{THF}$ & $\mathrm{P} 1$ & T3 & $0 / 0$ \\
\hline
\end{tabular}

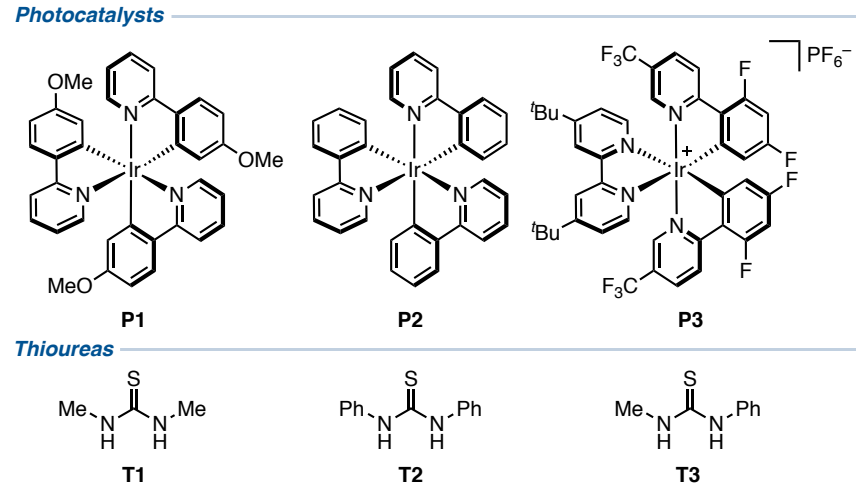

aConditions: 1A (0.10 mmol), zirconocene (5.0 mol \%), photocatalyst (3.0 mol \%), thiourea (60 mol \%), 1,4-CHD (3.0 equiv),
$\mathrm{PhCF}_{3}(2.0 \mathrm{~mL}), 35^{\circ} \mathrm{C}, 12$ h. NMR Yield. ${ }^{b}$ In toluene. ${ }^{c}$ In THF. $d$ With MS4Å (50 mg). e Without irradiation.

To this end, we set out to investigate a catalytic protocol for the ring opening of epoxides using zirconocene with photocatalysts even though Zr (III) has been scarcely utilized in organic synthesis. ${ }^{26-32}$ Since the reduction of zirconocene typically requires high reducing power $\left(-E_{1 / 2}\right.$ $\left(\mathrm{Cp}_{2} \mathrm{ZrCl}_{2}\right)=-1.85 \mathrm{~V}$ vs SCE$)^{33}$, we commenced with strongly reducing photocatalyst $\operatorname{Ir}[(4-\mathrm{MeO}) p p y]_{3}$ (P1, $E_{1 / 2}$ $\operatorname{Ir}(\mathrm{IV}) / \operatorname{Ir}(\mathrm{III})^{*}=-1.95 \mathrm{~V}$ vs SCE in MeCN). ${ }^{34}$ Visible light irradiation of $\mathbf{1 A}$ in the presence of zirconocene dichloride, P1, and 1,4-cyclohexadiene (CHD) resulted in no product formation (Table 1, entry 1 ). A change to $\mathrm{Cp}_{2} \mathrm{Zr}(\mathrm{OTf})_{2} \cdot \mathrm{THF}$ did not furnish either of the alcohols (Table 2, entry 2). Gratifyingly, we found that addition of dimethylthiourea (T1) furnished the desired alcohol 1B in 34\% yield along with the isomeric alcohol $\mathbf{1 C}$ in $7 \%$ yield (Table 1, entry 3 ). Further screening revealed that the combination of 1 methyl-3-phenyl thiourea (T3) and $\mathrm{PhCF}_{3}$ facilitates the reaction with a suppressed amount of $1 \mathrm{C}$ (Table 1, entries 4-7). Applying $\operatorname{Ir}(\text { ppy) })_{3}$ (P2, $E_{1 / 2} \operatorname{Ir}(\mathrm{IV}) / \operatorname{Ir}(\mathrm{III})^{*}=-1.88 \mathrm{~V}$ vs $\mathrm{SCE}$ in $\mathrm{MeCN}^{34}$ or $\operatorname{Ir}\left[\left(\mathrm{dFCF}_{3} \mathrm{ppy}\right)_{2}(\mathrm{dtbbpy})\right] \mathrm{PF}_{6}$ (P3, $E_{1 / 2}$ $\operatorname{Ir}(\mathrm{IV}) / \mathrm{Ir}(\mathrm{III})^{*}=-1.21 \mathrm{~V}$ vs SCE in MeCN ${ }^{35}$ diminished the yield of 1B (Table 1, entries 8 and 9). Although other zirconocenes were not effective, the addition of molecular sieves slightly increased the yield (Table 1, entries 10-12). Control experiments excluding zirconocene, photocatalyst, and light provided essentially no product (Table 1, entries 13-15).

With the optimized conditions in hand, we next evaluated the substrate scope of this protocol (Scheme 1). First, we examined terminal epoxides with a variety of functional groups. In addition to phenyl (1A), $p$-methoxyphenyl (1B), alkyl (1C), ester (1D-1F), benzyl and silyl ether (1G and 1H), and chloride (1I) were accommodated to afford the corresponding secondary alcohols with high yield and regioselectivity. Electronically diverse substituents on aromatic rings (1J-10) were all tolerated. Protected prolinol derivatives (1P-1R), as well as sulfonamide (1S), were found to be suitable substrates. We next evaluated di- and trisubstituted epoxides. The present protocol converted 1,1-disubstituted epoxides $\mathbf{1 T}$ and $\mathbf{1 U}$ to more-substituted alcohols with retained regioselectivity, whereas ring opening of epoxide $\mathbf{1 V}$ afforded $41 \%$ yield of $\mathbf{2 V}$ and $\mathbf{3 V}$ in total with considerable amount of cyclic product $4 \mathrm{~V}$ suggesting the addition of a primary radical to the aromatic ring. Spiro epoxides (1W-1Z) uniformly furnished the desired alcohol, although incorporation of bulky adamantane (1AA) considerably reduced regioselectivity. 1,2-Disubstituted epoxides with a variety of ring sizes were well-tolerated (1AB1AF). Interestingly, ring opening of dibenzyl ether $\mathbf{1 A G}$ produced alcohol 2AG along with benzylidene acetal 4AG obtained in the previous titanocene-mediated ring opening via 1,5-hydrogen atom transfer (HAT). ${ }^{36}$ In addition to trisubstituted epoxides (1AH-1AJ), natural product-derived epoxides (1AK-1AN) could also be readily opened in good yields. 


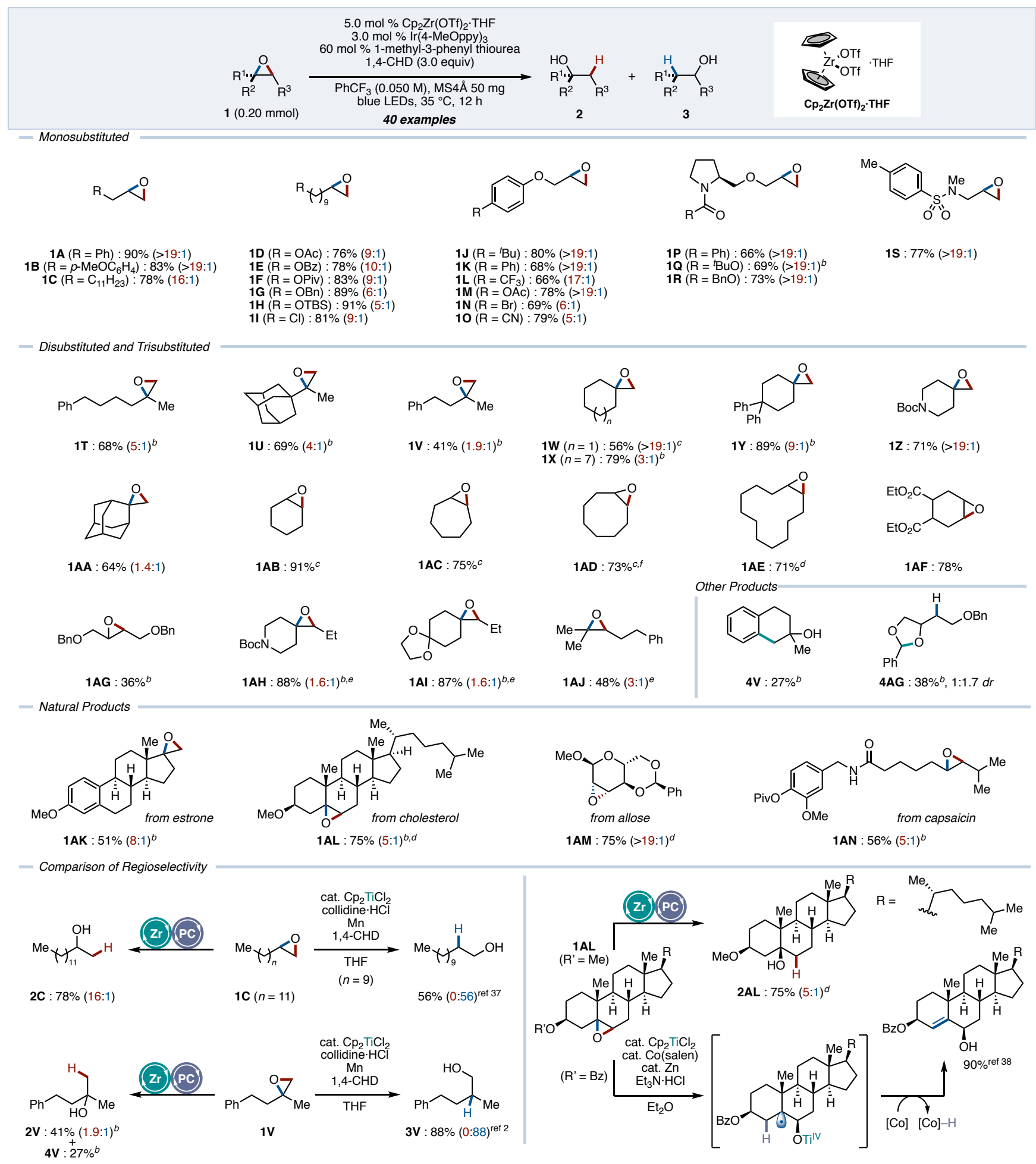

${ }^{a}$ Conditions: 1 (0.20 mmol), Cp2Zr(OTf) $2 \cdot \operatorname{THF}$ (5.0 mol \%), Ir(4-MeOppy) 3 (3.0 mol \%), T3 (60 mol \%), 1,4-CHD (3.0 equiv), PhCF 3 $(4.0 \mathrm{~mL}), 35^{\circ} \mathrm{C}, 12 \mathrm{~h}$. All data are the average of two experiments. Unless otherwise noted, yields are for purified material of inseparable alcohols and isomeric ratios are determined by ${ }^{1} \mathrm{H}$ NMR analysis of crude reaction mixture. ${ }^{b}$ Isomeric ratios are for isolated material. $c$ Yields and isomeric ratios were determined by GC analysis due to volatility. ${ }^{d} 10.0 \mathrm{~mol} \%$ of $\mathrm{Cp}_{2} \mathrm{Zr}(\mathrm{OTf})_{2} \cdot \mathrm{THF}$ was used. e4,4-Dimethyl-2-imidazolidinethione was used instead of T3. Reactions were performed for $24 \mathrm{~h}$. See the SI for details.

Comparisons to the analogous precedents performed by titanocene highlighted the reverse reactivity in ring opening. As mentioned above, ring opening of $1 \mathrm{C}$ provided 2C with high regioselectivity, which was contrasting to the previous study with titanocene. ${ }^{37}$ Similarly, the ring opening of $1 \mathrm{~V}$ afforded tertiary and primary alcohols along with $\mathbf{4 V}$ in our conditions, whereas primary alcohol $\mathbf{3 V}$ is preferably obtained with titanocene. ${ }^{2}$ While our protocol con- 
verted $\mathbf{1 A L}$ to $\mathbf{2 A L}$, ring opening of a similar cholesterol ( $\mathrm{R}^{\prime}$ $=\mathrm{Bz}$ ) with titanocene furnished a tertiary radical, and the adjacent hydrogen was abstracted by a cobalt catalyst leading to an allylic alcohol. ${ }^{38}$ These contrasting results demonstrated that our catalytic protocol is complementary to conventional methods with titanocene.

To gain insights into the reaction mechanism, ring opening of cyclopropyl-bearing epoxide $\mathbf{1 A O}$ was conducted (Figure 2A). Treatment of this probe with the optimized conditions afforded the allylic alcohol 5AO, suggesting a C$\mathrm{C}$ bond cleavage via a cyclopropylcarbinyl radical. The same product was obtained in Oshima's conditions where $\mathrm{Zr}(\mathrm{III})$ was prepared from $\mathrm{Cp}_{2} \mathrm{Zr}(\mathrm{H}) \mathrm{Cl}^{2}{ }^{29}$ Furthermore, intramolecular radical addition could be achieved with an epoxy ester (1AP; Figure 2B). These observations, as well as formation of $\mathbf{4 V}$ and $\mathbf{4 A G}$, are in agreement with the $\mathrm{C}-\mathrm{O}$ bond cleavage proceeding via a radical pathway.

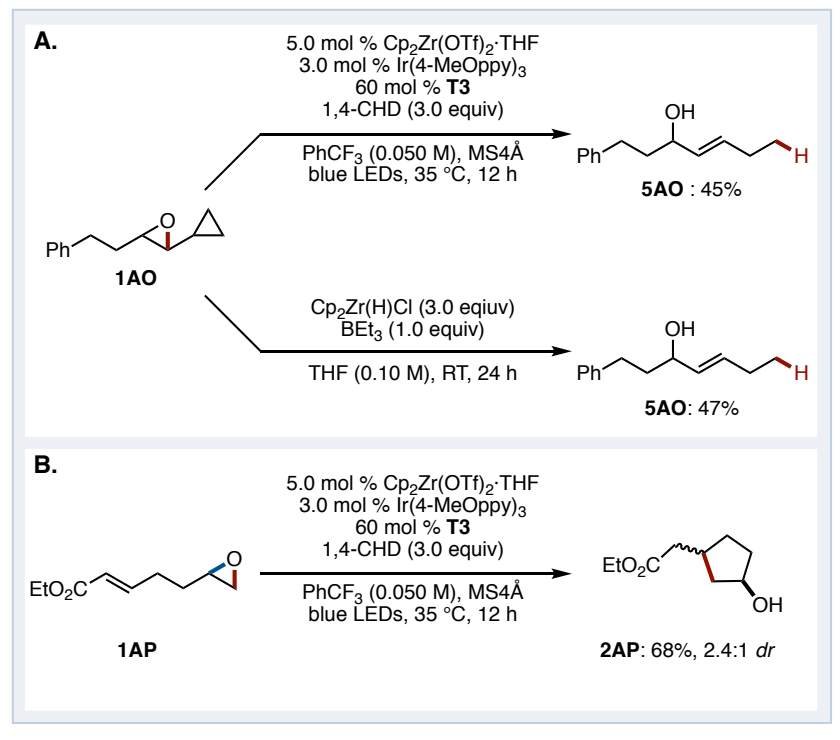

Figure 2 (A) Radical clock experiment. (B) Intramolecular radical addition.

Next, we performed DFT calculations to reveal the energy profile of ring opening and HAT based on a model reaction of 1,1-dimethyl oxirane and metallocene triflates (III) $(\mathrm{M}=\mathrm{Ti}$ or $\mathrm{Zr}$ ) (Figure $3 \mathrm{~A})$. The reaction proceeds from complex I via the respective transition state (TS-Ia and TSIb) to afford the corresponding ring-opened forms (IIa and IIb). The resulting radicals would then undergo HAT with CHD to be converted to alcohols (IIIa and IIIb). The energy profile with primary (purple) and tertiary (aqua) radical with titanocene, and primary (red) and tertiary (blue) radical with zirconocene are shown in Figure 3A. In accord with our design hypothesis, activation free energies of ring opening $\Delta G_{\text {TS-I }}$ with zirconocene $(7.81$ and $6.24 \mathrm{kcal} / \mathrm{mol}$, respectively) are remarkably lower than those of titanocene $\left(22.14\right.$ and $14.95 \mathrm{kcal} / \mathrm{mol}$, respectively). ${ }^{39}$ Similarly, $\Delta \Delta G_{\text {TS-I }}$ with zirconocene $(1.57 \mathrm{kcal} / \mathrm{mol})$ is much smaller than that of titanocene $(7.19 \mathrm{kcal} / \mathrm{mol})$. This significant difference can be accounted for by the consequence of shifting the TS of ring opening earlier, which would reduce the influence of the thermodynamic stability between the resulting primary and tertiary radicals at the TS. Structures of TS-1 provide further insight into shifting the TS of ring opening. The scissile $\mathrm{C}-\mathrm{O}$ bonds in $\mathbf{Z r}$-TS-I are shorter than that of Ti-TS-I by approximately $0.2 \AA$, suggesting that Zr-TS-I are more reactant-like. The spin density on the evolving carbon radical in Zr-TS-Ia (0.39) and Zr-TSIb (0.29) was smaller than that in Ti-TS-Ia (0.57) and TiTS-Ib (0.42). These structural features are consistent with more reactant-like $\mathbf{Z r}$-TS.

DFT calculations indicated that ring opening with titanocene was endothermic, which is consistent with a previous study. ${ }^{36}$ Since titanocene-bounded tertiary radical IIb is more stable than the corresponding primary radical IIa by $6.82 \mathrm{kcal} / \mathrm{mol}$, ring opening under thermodynamic control results in a large abundance of IIb which undergoes HAT to afford IIIb as a major product. In contrast, ring opening with zirconocene was found to be extremely exothermic and practically irreversible. By means of computational studies to provide a rough picture of the energy profile, $\mathbf{Z r}$ TS-Ia was calculated to be less stable than Zr-TS-IIb. This discrepancy with our results prompted further investigation into the identity of the zirconocene complex.

In a possible scenario to explain the regioselectivity, ring opening proceeds via an epoxide-zirconocene-thiourea complex. ${ }^{40-42}$ In this case, $\Delta G_{\text {T-Ia }}$ might be lower than $\Delta$ $G_{\text {TS-lb, }}$ which is opposite to the calculated results with the zirconocene triflate complex. Diffusion-ordered NMR spectroscopy (DOSY) measurements evidently demonstrated that thiourea interacts with zirconocene (Figure 3B). In this experiment, tetramethylthiourea was used to facilitate NMR analysis. A 1:1 mixture of zirconocene and thiourea furnished several new signals in ${ }^{1} \mathrm{H}$ NMR. The diffusion coefficient for the newly generated signals $\left(D=1.44 \times 10^{-9}\right.$ $\left.\mathrm{m}^{2} / \mathrm{s}\right)$ was smaller than that of zirconocene $\left(D=1.55 \times 10^{-9}\right.$ $\mathrm{m}^{2} / \mathrm{s}$ ) and completely different from that of parent thiourea $\left(D=3.45 \times 10^{-9} \mathrm{~m}^{2} / \mathrm{s}\right)$, suggesting the interaction of zirconocene and thiourea. Thiourea probably interacts with not only $\operatorname{Zr}(\mathrm{IV})$ but also $\operatorname{Zr}(\mathrm{III})$ species since regioselectivity of the ring opening was influenced by the selected thiourea. Furthermore, Stern-Volmer analysis indicated that quenching of the excited photocatalyst by the zirconocene thiourea complex (see the SI for details) 
A. Energy Diagram of Epoxide Ring Opening and Hydrogen Atom Abstraction with Metallocene(III)
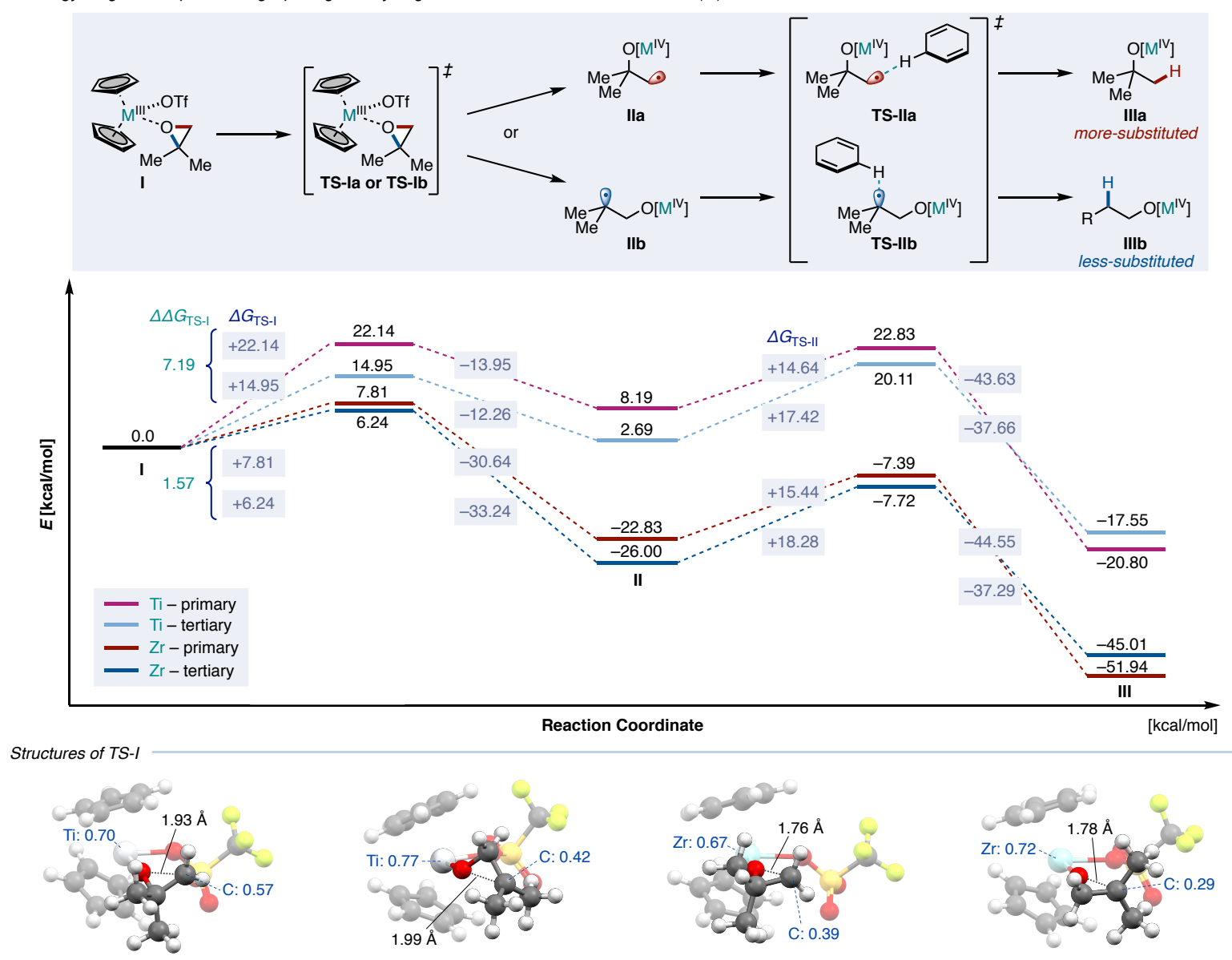

Ti-TS-la

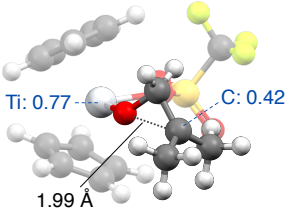

Ti-TS-|b

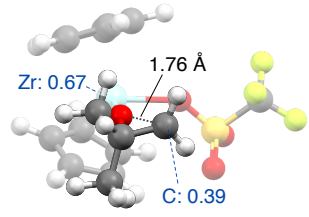

Zr-TS-la

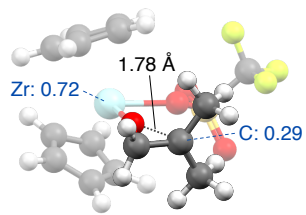

Zr-TS-Ib

B. Interaction of Zirconocene and Thiourea

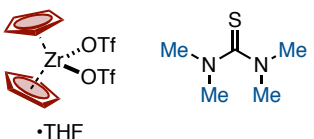
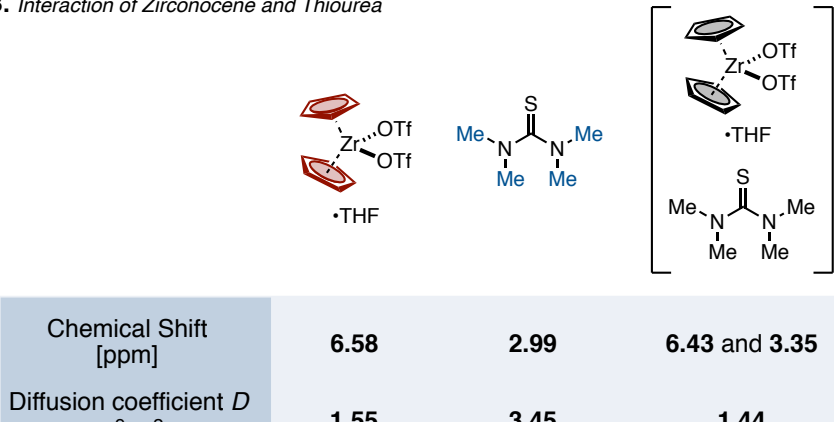

6.58

2.99

6.43 and 3.35

$\left[10^{-9} \mathrm{~m}^{2} / \mathrm{s}\right]$

1.55

3.45

1.44

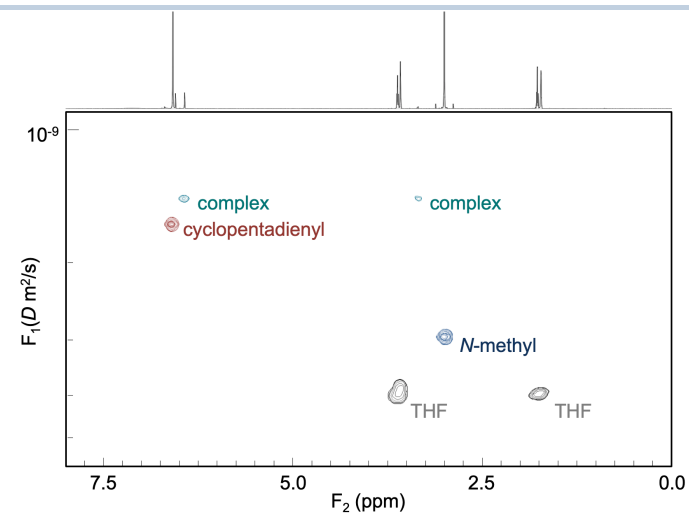

Figure 3. Mechanistic studies. (A) DFT calculations for the ring opening and HAT of asymmetric epoxide with metallocene triflate (III). Potential energies of transition states (TS) and intermediates (I, II, and III) in ring opening and HAT (B3LYP/6$\left.31+\mathrm{G}(\mathrm{d}, \mathrm{p}) / / \mathrm{B} 3 \mathrm{LYP} / \mathrm{def}_{2} \mathrm{SVP}\right)$ are shown. Each $\Delta G$ value between two structures is shown in the light blue box. All energies are given in $\mathrm{kcal} / \mathrm{mol}$. (B) A DOSY spectrum for a 1:1 mixture of $\mathrm{Cp}_{2} \mathrm{Zr}(\mathrm{OTf})_{2} \cdot \mathrm{THF}$ and tetramethylthiourea in THF- $d_{8}$ at $25{ }^{\circ} \mathrm{C}$. Chemical shifts and diffusion coefficients of zirconocene, thiourea, and the mixture are shown respectively.

Given these experimental results, a possible mechanism is depicted in Figure 4. The reaction could be initiated by reduction of zirconocene by an excited photocatalyst to furnish $\operatorname{Zr}($ III) and triflate. Coordination between $\mathrm{Zr}$ (III) and epoxide could result in $\mathrm{C}-\mathrm{O}$ bond homolysis to afford a carbon radical, which abstracts hydrogen from 1,4-CHD. The cyclohexadienyl radical undergo oxidation by IrIV $^{\mathrm{IV}}$ catalyst $\left(E_{1 / 2} \operatorname{Ir}(\mathrm{IV}) / \operatorname{Ir}(\mathrm{III})=+0.70 \mathrm{~V}\right.$ vs SCE in MeCN $){ }^{34}$ The resulting cation and triflate would work as a Brønsted acid to protonate zirconocene alkoxide to complete the catalytic cycle, with release of the desired alcohol and benzene. ${ }^{17}$ 


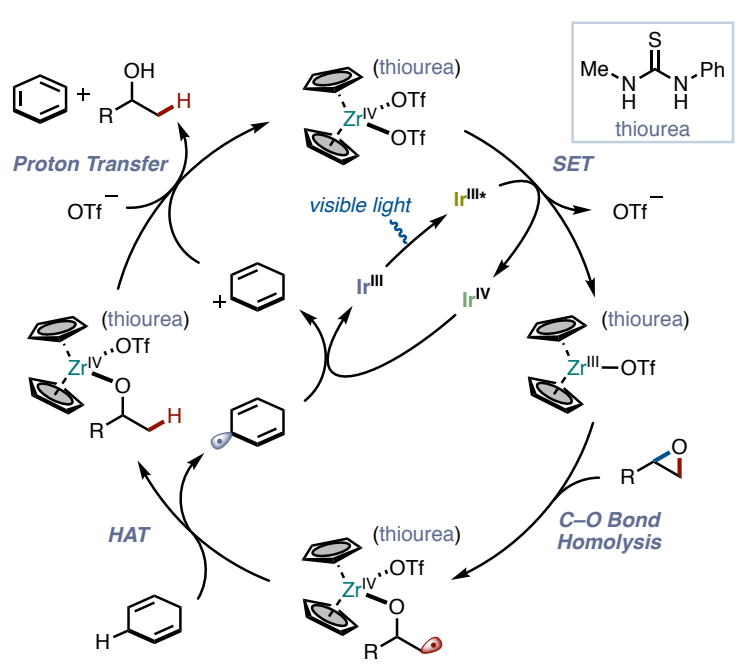

Figure 4. Proposed catalytic cycle.

In summary, we succeeded in the development of a zirconocene-photoredox cooperative catalysis for the ring opening of epoxide, exhibiting reverse regioselectivity compared to titanocene-mediated reactions. To our knowledge, this is the first example that harnesses a radical intermediate generated by $\mathrm{C}-\mathrm{O}$ homolysis of epoxide using zirconocene. ${ }^{43-45}$ Exploring transformations with this new cooperative catalysis and further mechanistic studies are currently underway in our laboratory.

\section{ASSOCIATED CONTENT}

\section{Supporting Information}

The Supporting Information is available free of charge on the ACS Publications website.

Experimental procedures, additional experimental results, computational study, and compound characterization (PDF)

\section{AUTHOR INFORMATION}

\section{Corresponding Author}

* Eisuke Ota - Department of Applied Chemistry, Waseda University, 513 Wasedatsurumakicho, Shinjuku, Tokyo 162-0041, Japan; orcid.org/0000-0002-3436-9768; Email: eo-

ta@aoni.waseda.jp

* Junichiro Yamaguchi - Department of Applied Chemistry, Waseda University, 513 Wasedatsurumakicho, Shinjuku, Tokyo 162-0041, Japan;

orcid.org/0000-0002-3896-5882; Email: junyamagu-

chi@waseda.jp

\section{Author}

Kazuhiro Aida - Department of Applied Chemistry, Waseda University, 513 Wasedatsurumakicho, Shinjuku, Tokyo 1620041, Japan

Marina Hirao - Department of Applied Chemistry, Waseda University, 513 Wasedatsurumakicho, Shinjuku, Tokyo 1620041, Japan

Aiko Funabashi - Department of Applied Chemistry, Waseda University, 513 Wasedatsurumakicho, Shinjuku, Tokyo 1620041, Japan
Natsuhiko Sugimura - Materials Characterization Central Laboratory, Waseda University, 3-4-1 Ohkubo, Shinjuku, Tokyo 169-8555, Japan

Notes

The authors declare no competing financial interest.

\section{ACKNOWLEDGMENT}

This work was supported by JSPS KAKENHI Grant Number JP19H02726 (to J.Y.), JP20K15290 (to E.O.), Sumitomo Foundation (to E.0.), Fukuoka Naohiko Memorial Foundation (to E.O.), Satomi Foundation (to E.O.), JXTG Nippon Oil \& Energy Corporation (to E.O.). This work was partly supported by JST ERATO Grant Number JPMJER1901 (to J.Y.). We thank Prof. Robert Knowles for helpful comments. Materials Characterization Central Laboratory in Waseda University is acknowledged for the support of HRMS measurement. A part of computations was performed at the Research Center for Computational Science, Okazaki, Japan.

\section{REFERENCES}

(1) Nugent, W. A.; RajanBabu, T. V. Transition-Metal-Centered Radicals in Organic Synthesis. Titanium(III)-Induced Cyclization of Epoxy Olefins. J. Am. Chem. Soc. 1988, 110, 8561-8562.

(2) The first example of catalytic reaction, see: Gansäuer, A.; Pierobon, M.; Bluhm, H. Catalytic, Highly Regio- and Chemoselective Generation of Radicals from Epoxides: Titanocene Dichloride as an Electron Transfer Catalyst in Transition Metal Catalyzed Radical Reactions. Angew. Chem., Int. Ed. 1998, 37, 101-103.

(3) Krische, M. J. Radicals in Synthesis III; Heinrich, M., Gansäuer, A., Eds.; Topics in Current Chemistry; Springer Berlin Heidelberg: Berlin, Heidelberg, 2012; Vol. 320.

(4) Rosales, A.; Rodríguez-García, I.; Muñoz-Bascón, J.; RoldanMolina, E.; Padial, N. M.; Morales, L. P.; García-Ocaña, M.; Oltra, J. E. The Nugent Reagent: A Formidable Tool in Contemporary Radical and Organometallic Chemistry. Eur. J. Org. Chem. 2015, 2015, 4567-4591.

(5) Castro Rodríguez, M.; Rodríguez García, I.; Rodríguez Maecker, R. N.; Pozo Morales, L.; Oltra, J. E.; Rosales Martínez, A. Cp2 TiCl: An Ideal Reagent for Green Chemistry? Org. Process Res. Dev. 2017, 21, 911-923.

(6) McCallum, T.; Wu, X.; Lin, S. Recent Advances in Titanium Radical Redox Catalysis. J. Org. Chem. 2019, 84, 14369-14380.

(7) Martínez, A. R.; Morales, L. P.; Ojeda, E. D.; Rodríguez, M. C.; Rodríguez-García, I. The Proven Versatility of $\mathrm{Cp}_{2} \mathrm{TiCl}$. J. Org. Chem. 2021, 86, 1311-1329.

(8) Zhao, Y.; Weix, D. J. Nickel-Catalyzed Regiodivergent Opening of Epoxides with Aryl Halides: Co-Catalysis Controls Regioselectivity. J. Am. Chem. Soc. 2014, 136, 48-51.

(9) Morcillo, S. P.; Miguel, D.; Campaña, A. G.; Álvarez de Cienfuegos, L.; Justicia, J.; Cuerva, J. M. Recent Applications of $\mathrm{Cp}_{2} \mathrm{TiCl}$ in Natural Product Synthesis. Org. Chem. Front. 2014, 1, 15-33.

(10) Zweig, J. E.; Kim, D. E.; Newhouse, T. R. Methods Utilizing First-Row Transition Metals in Natural Product Total Synthesis. Chem. Rev. 2017, 117, 11680-11752.

(11) Padial, N. M.; Roldan-Molina, E.; Rosales, A.; Álvarez-Corral, M.; Rodríguez-García, I.; Muñoz-Dorado, M.; Oltra, J. E. Stereoselective Synthesis of Natural Products Promoted by Titanocene(III). In Studies in Natural Products Chemistry; Atta-ur-Rahman, Ed.; Elsevier B.V., 2018; Vol. 55, pp 31-71.

(12) Zhang, Z.; Richrath, R. B.; Gansäuer, A. Merging Catalysis in Single Electron Steps with Photoredox Catalysis-Efficient and Sustainable Radical Chemistry. ACS Catal. 2019, 9, 3208-3212.

(13) Lin, S.; Chen, Y.; Li, F.; Shi, C.; Shi, L. Visible-Light-Driven Spirocyclization of Epoxides via Dual Titanocene and Photoredox Catalysis. Chem. Sci. 2020, 11, 839-844. 
(14) Parasram, M.; Shields, B. J.; Ahmad, O.; Knauber, T.; Doyle, A. G. Regioselective Cross-Electrophile Coupling of Epoxides and (Hetero)aryl Iodides via Ni/Ti/Photoredox Catalysis. ACS Catal. 2020, 10, 5821-5827.

(15) Gansäuer, A.; Barchuk, A.; Keller, F.; Schmitt, M.; Grimme, S.; Gerenkamp, M.; Mück-Lichtenfeld, C.; Daasbjerg, K.; Svith, H. Mechanism of Titanocene-Mediated Epoxide Opening through Homolytic Substitution. J. Am. Chem. Soc. 2007, 129, 1359-1371.

(16) Gansäuer, A.; Fleckhaus, A.; Lafont, M. A.; Okkel, A.; Kotsis, K.; Anoop, A.; Neese, F. Catalysis via Homolytic Substitutions with $\mathrm{C}-\mathrm{O}$ and Ti-O Bonds: Oxidative Additions and Reductive Eliminations in Single Electron Steps. J. Am. Chem. Soc. 2009, 131, 1698916999.

(17) Klare, S.; Gordon, J. P.; Gansäuer, A.; RajanBabu, T. V.; Nugent, W. A. The Reaction of $\beta, \gamma$-epoxy Alcohols with Titanium(III) Reagents. A Proposed Role for Intramolecular Hydrogen Bonding. Tetrahedron 2019, 75, 130662

(18) Fernández-Mateos, A.; Madrazo, S. E.; Teijón, P. H.; González, R. R. Titanocene-Promoted Intermolecular Couplings of Epoxides with Nitriles. An Easy Access to $\beta$-Hydroxyketones. J. Org. Chem. 2009, 74, 3913-3918.

(19) Barrero, A. F.; Del Moral, J. F. Q.; Sánchez, E. M.; Arteaga, J. F. Regio- and Diastereoselective Reductive Coupling of Vinylepoxides Catalyzed by Titanocene Chloride. Org. Lett. 2006, 8, 669672.

(20) Parrish, J. D.; Daniel Little, R. Preparation of $\alpha$-C-Glycosides from Glycals. Org. Lett. 2002, 4, 1439-1442.

(21) Several cross-coupling reactions with aryl halides via titanocene-mediated ring opening of epoxides afford more-substituted alcohols, see: references 8 and 14 .

(22) Bell, R. P. The Theory of Reactions Involving Proton Transfers. Proc. R. Soc. A 1936, 154, 414-429.

(23) Evans, M. G.; Polanyi, M. Further Considerations on the Thermodynamics of Chemical Equilibria and Reaction Rates. Trans. Faraday Soc. 1936, 32, 1333-1360.

(24) Hammond, G. S. A Correlation of Reaction Rates. J. Am. Chem. Soc. 1955, 77, 334-338.

(25) Lappert, M. F.; Patil, D. S.; Pedley, J. B. Standard Heats of Formation and M-C Bond Energy Terms for Some Homoleptic Transition Metal Alkyls MR N. J. Chem. Soc., Chem. Commun. 1975, 20, 830-831.

(26) Titanium and Zirconium in Organic Synthesis; Marek, I., Ed.; Wiley-VCH Verlag GmbH \& Co. KGaA, 2002.

(27) Lancaster, S. J. 4.07 - Complexes of Zirconium and Hafnium in Oxidation State iii. In Comprehensive Organometallic Chemistry III; Compounds of Groups 3 to 4 and the f elements, Vol. 4; Elsevier, 2007, pp 741-757.

(28) Barden, M. C.; Schwartz, J. Stereoselective "Pinacol” Coupling of 2,3-O-Isopropylidene-D-Glyceraldehyde. J. Org. Chem. 1997, 62, $7520-7521$.

(29) Fujita, K.; Yorimitsu, H.; Oshima, K. Development of Radical Reactions with Zirconocene Complexes as Electron Transfer Reagents. Bull. Chem. Soc. Jpn. 2004, 77, 1727-1736.

(30) Lakshmi Kantam, M.; Aziz, K.; Likhar, P. R. Zirconocene Dichloride-Catalyzed Pinacol Coupling of Aromatic Aldehydes and Ketones. Synth. Commun. 2006, 36, 1437-1445.

(31) Alpers, D.; Hoffmann, F.; Brasholz, M. Visible-Light Photolysis of Allyl Zirconocenes: A Photoinduced Three-Component Radical (4+2)-Cyclization-Allylation Reaction. Synlett 2017, 28, 919-923.

(32) Gao, Y.; Yang, C.; Bai, S.; Liu, X.; Wu, Q.; Wang, J.; Jiang, C.; Qi, X. Visible-Light-Induced Nickel-Catalyzed Cross-Coupling with Alkylzirconocenes from Unactivated Alkenes. Chem 2020, 6, 675688.

(33) Zachmanoglou, C. E.; Docrat, A.; Bridgewater, B. M.; Parkin, G.; Brandow, C. G.; Bercaw, J. E.; Jardine, C. N.; Lyall, M.; Green, J. C.; Keister, J. B. The Electronic Influence of Ring Substituents and Ansa Bridges in Zirconocene Complexes as Probed by Infrared Spectroscopic, Electrochemical, and Computational Studies. J. Am. Chem. Soc. 2002, 124, 9525-9546.
(34) Nacsa, E. D.; MacMillan, D. W. C. Spin-Center Shift-Enabled Direct Enantioselective $\alpha$-Benzylation of Aldehydes with Alcohols. J. Am. Chem. Soc. 2018, 140, 3322-3330.

(35) Lowry, M. S.; Goldsmith, J. I.; Slinker, J. D.; Rohl, R.; Pascal, R. A.; Malliaras, G. G.; Bernhard, S. Single-Layer Electroluminescent Devices and Photoinduced Hydrogen Production from an Ionic Iridium(III) Complex. Chem. Mater. 2005, 17, 5712-5719.

(36) Funk, P.; Richrath, R. B.; Bohle, F.; Grimme, S.; Gansäuer, A. Oxidation Under Reductive Conditions: From Benzylic Ethers to Acetals with Perfect Atom-Economy by Titanocene(III) Catalysis. Angew. Chem., Int. Ed. 2021, 60, 5482-5488.

(37) Gansäuer, A.; Bluhm, H.; Pierobon, M. Emergence of a Novel Catalytic Radical Reaction: Titanocene-Catalyzed Reductive Opening of Epoxides. J. Am. Chem. Soc. 1998, 120, 12849-12859.

(38) Ye, K.-Y.; McCallum, T.; Lin, S. Bimetallic Radical Redox-Relay Catalysis for the Isomerization of Epoxides to Allylic Alcohols. J. Am. Chem. Soc. 2019, 141, 9548-9554.

(39) The activation free energy for similar trisubstituted epoxides affording tertiary radicals using $\mathrm{Cp}_{2}$ TiOMs were calculated as 13.9 or $14.8 \mathrm{kcal} / \mathrm{mol}$, see reference 36 .

(40) Zirconocene complexes with oxadiazolethione and thiohydantoin was reported, see: Srivastava, V.; Pandey, O. P.; Sengupta, S. K.; Tripathi, S. C. Syntheses and Spectroscopic Studies of Monoand Di- Cyclopentadienylzirconium(IV) Derivatives with Heterocyclic Thiones. J. Organomet. Chem. 1985, 279, 395-402.

(41) Zirconocene complexes with guanidine were reported, see: Fernández-Galán, R.; Navarro, J.-A.; Carrillo-Hermosilla, F.; Antiñolo, A.; López-Solera, I.; Villaseñor, E. Preparation and Structural Studies of Non-Symmetric Guanidinate-Supported Zirconium Complexes. Aust. J. Chem. 2014, 67, 1063-1070.

(42) Thioureas are known to form a complex with titanocene to tune its redox potential while tetramethylthiourea also works as an effective additive in this study, see; Liedtke, T.; Spannring, P.; Riccardi, L.; Gansäuer, A. Mechanism-Based Condition Screening for Sustainable Catalysis in Single-Electron Steps by Cyclic Voltammetry. Angew. Chem., Int. Ed. 2018, 57, 5006-5010.

(43) C-O bond cleavage of epoxide using zirconocene in ionic mechanism, see: Hanzawa, Y.; Kiyono, H.; Tanaka, N.; Taguchi, T. New " $\mathrm{Cp}_{2} \mathrm{Zr}$ "-Mediated Ring Transformations of 2Vinylheterocycles to Carbocycles. Tetrahedron Lett. 1997, 38, 4615-4618. See also references 44 and 45.

(44) Wipf, P.; Xu, W.; Kim, H.; Takahashi, H. ZirconoceneCatalyzed Epoxy Ester - Ortho Ester Rearrangement: A New Method for the Protection of Polyfunctionalized Carboxylic Acids and the Asymmetric Synthesis of Ortho Esters. Tetrahedron 1997, $53,16575-16596$

(45) Blum, S. A.; Rivera, V. A.; Ruck, R. T.; Michael, F. E.; Bergman R. G. Synthetic and Mechanistic Studies of Strained Heterocycle Opening Reactions Mediated by Zirconium(IV) Imido Complexes. Organometallics 2005, 24, 1647-1659. 


$$
\text { less-substituted }
$$

